\begin{tabular}{c|c} 
Revista Electrónica Teoría de la Educación. \\
Educación y Cultura en la Sociedad de la Información.
\end{tabular}

\title{
METODOLOGÍA COMUNICATIVA CRÍTICA: TRANSFORMACIONES Y CAMBIOS EN EL S. XXI*
}

Resumen: Los análisis educativo-sociales que se llevan a cabo sobre la actual sociedad descubren valores cada vez más dialógicos. Para analizar esta sociedad se hace necesario disponer de una metodología que responda a ese carácter cada vez más dialógico y complejo. La metodología comunicativa crítica responde a esta nueva situación, contribuyendo a la superación de desigualdades educativas y sociales y fomentando la inclusión social de los colectivos que se encuentran en situaciones más desfavorecidas. En este artículo presentamos cómo a través de la metodología comunicativa crítica podemos llevar a cabo análisis de problemáticas educativas y sociales que muy difícilmente podrían ser abordados desde metodologías más tradicionales. Para ello, introduciremos brevemente las principales características de la sociedad en la que nos encontramos, qué nuevos retos se nos plantean y cómo mediante la metodología comunicativa crítica podemos contribuir a superar situaciones de exclusión social.

Palabras clave: metodología comunicativa crítica, inclusión social, giro dialógico.

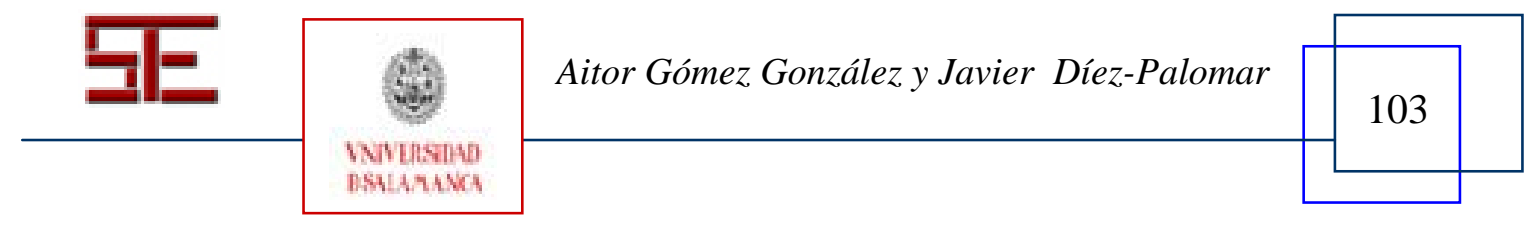




\section{CRITICAL COMMUNICATIVE METHODOLOGY: TRANSFORMATIONS AND CHANGES IN THE S. XXI}

Abstract: The educational and social analysis conducted on the current society increasingly discovers more dialogical values. To analyze this society a methodology that meets this increasingly complexity and dialogic character is needed. The critical communicative methodology responds to this new situation, helping to overcome educational and social inequalities and promoting social inclusion of groups who are in disadvantaged situations. In this article we present how through the critical communicative methodology we can carry out analysis of social and educational problems which can hardly be approached from more traditional methodologies. For this, we introduce briefly the main features of the society in which we find, the new challenges facing us and how the critical communicative methodology can help to overcome situations of social exclusion.

Keywords: critical communicative methodology, social inclusion, dialogic turn

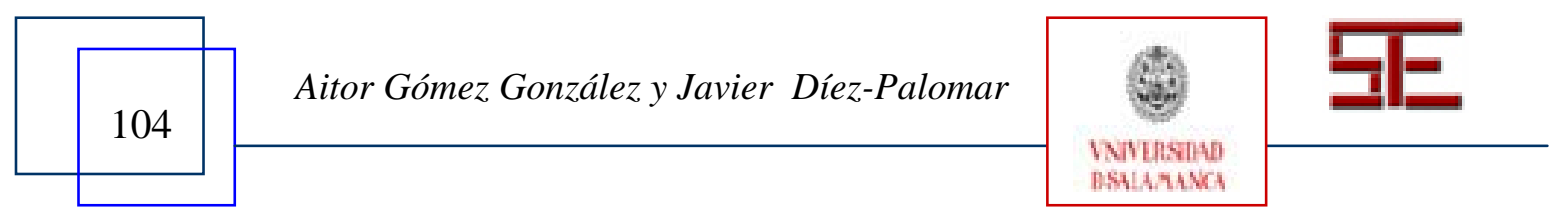




\begin{tabular}{c|c} 
Revista Electrónica Teoría de la Educación. \\
Educación y Cultura en la Sociedad de la Información.
\end{tabular}

\title{
METODOLOGÍA COMUNICATIVA CRÍTICA: TRANSFORMACIONES Y CAMBIOS EN EL S. XXI
}

\author{
Aitor Gómez González. \\ Universitat Rovira i Virgili. \\ aitor.gomez@urv.cat \\ Javier Díez-Palomar. \\ Universitat Autònoma de Barcelona. \\ Javier.diez@uab.cat
}

\section{1.- INTRODUCCIÓN: EL GIRO DIALÓGICO}

Actualmente las personas que se dedican a hacer análisis de los cambios que acontecen en nuestra sociedad hablan de que se ha producido un cambio social de amplio calado que denominan "giro dialógico". Esta transformación va más allá de las tendencias de análisis sociales (análogo al giro "lingüístico" que se produjo en las ciencias sociales durante los años setenta y ochenta del siglo pasado). Se trata de una transformación que tiene repercusión en la vida cotidiana de todos nosotros y nosotras; y a través del diálogo y la inclusión de las voces de personas que tradicionalmente habían sido excluidas del ámbito académico, está produciendo también una revolución por lo que respecta a las perspectivas metodológicas y teóricas de trabajo académico (Flecha, Gómez y Puigvert, 2001).

¿En qué consiste este "giro dialógico" de la sociedad? Décadas atrás, las relaciones que teníamos por ejemplo en casa venían marcadas por el “cabeza” de familia. Él era quien tomaba las decisiones en base a relaciones de poder. Hoy en día, esas relaciones de poder se tambalean debido a que todas las personas quieren ser protagonistas a la hora de decidir. Las relaciones se tornan más dialógicas y se requiere del consenso para llegar a acuerdos (Aubert et al., 2008). Por otro lado, el impacto de la universalización del acceso a la información con recursos tales como Internet, da acceso igualitario a la información a cada vez más personas, que de esa manera también tienen más referentes y, por tanto, mayor criterio e información, para participar de manera crítica en la toma de decisiones importantes de sus propias vidas.

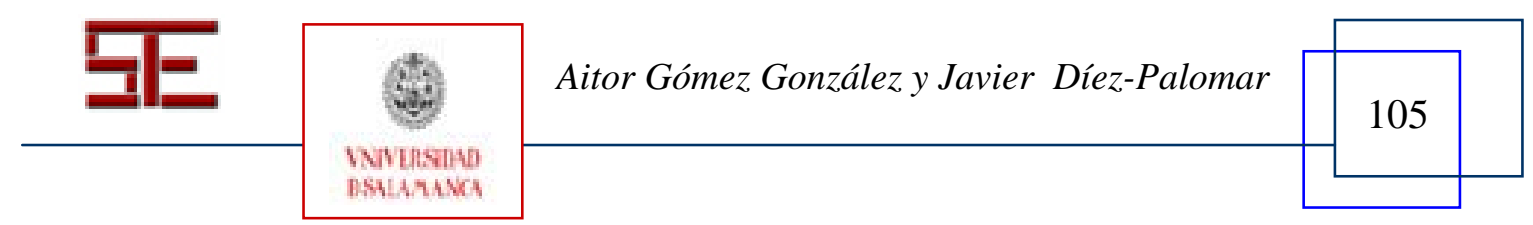


El giro dialógico es precisamente este cambio que se produce en nuestras casas y podemos ver también como aparece en otros ámbitos muy diversos. En los centros educativos se observa como la autoridad del profesorado también ha sufrido cambios, en el mundo empresarial la toma de decisiones depende cada vez más del diálogo y consenso. Algunos de los principales autores de las ciencias sociales (Beck, 1998; Flecha et al., 2001; Giddens, 1995; Habermas, 2001) analizan este proceso de incremento de diálogo en las decisiones que toman las personas a muy diferentes niveles.

La revolución tecnológica producida en el paso de la sociedad industrial a la sociedad de la información ha generado un aumento de las opciones a la hora de tomar decisiones que a su vez incide de manera directa en la sensación de riesgo que tienen las personas. Por ello, se incrementan los espacios y momentos en los cuales se pueden tomar decisiones mediante un diálogo igualitario, a través del cual cada persona expone argumentos en un plano de igualdad. Esta situación también incide en cómo se crean los conocimientos científicos, donde la figura del "experto" ha sufrido una profunda transformación, ya que no posee toda la información necesaria como para realizar aportaciones que sean útiles a todas las personas. Ahora las personas tienen más recursos para aportar argumentos y buscan espacios de diálogo para ello. Estamos frente a la desmonopolización del conocimiento experto (Beck et al., 1997).

Pero no sólo cambian las interacciones que se producen entre sistema y sujetos y entre los propios sujetos, sino la misma forma de elaborar las teorías. Actualmente las personas investigadoras incluyen el diálogo con los sujetos a la hora de explicar una realidad social compleja. Dado que este cambio social tiene como consecuencia importante que cada vez las personas somos más autoras de nuestras propias biografías (tenemos la tendencia a escoger entre múltiples opciones ${ }^{1} \mathrm{y}$ tomar decisiones), eso quiere decir que tenemos más capacidad de incidir sobre el mundo en el que vivimos. Por ese motivo, las teorías y las metodologías de trabajo que utilizamos tienen que re-pensarse para dar cabida a esta nueva situación y ajustarse a ella. Las perspectivas tradicionales de investigación, que tratan a las personas como "objetos de investigación”, a menudo lo que producen son esquemas que "corren paralelos a la realidad", pero que no sirven para entenderla, puesto que no incluyen las voces de esos actores que son quienes están (estamos) diseñando la realidad social a través de nuestras interacciones intersubjetivas.

La base ontológica de la que parte la metodología comunicativa crítica es una concepción de la realidad social como producto construido por las personas a través de nuestras interacciones. Entender la realidad desde este punto de vista exige el uso de técnicas metodológicas acordes, que sean válidas y fiables para describir los fenómenos sociales y educativos de nuestro mundo caracterizado por ese "giro dialógico". En los apartados siguientes se discutirá cómo la metodología comunicativa crítica es una nueva perspectiva de trabajo que responde a las exigencias metodológicas del momento en el que vivimos. A través de ejemplos concretos, se presentará la dimensión epistemológica que enmarca la metodología comunicativa crítica, y cómo se entienden (y redefinen) categorías clásicas de investigación como puedan ser "sujeto/objeto de investigación”, "validación de los resultados”, “veracidad”, “técnicas tanto de recogida como de análisis de

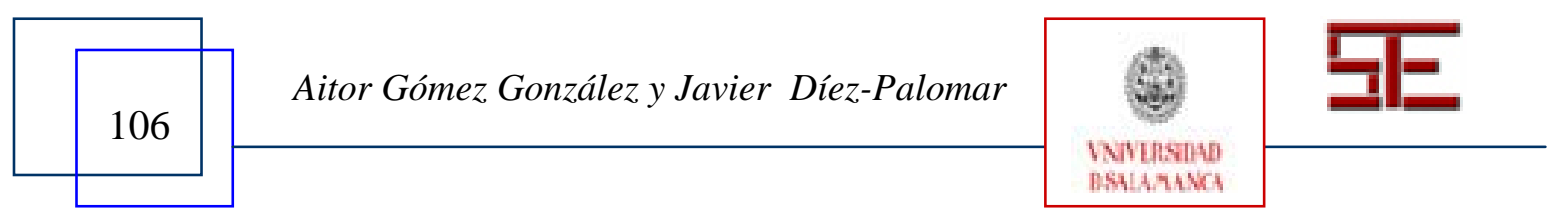




\begin{tabular}{c|c} 
Revista Electrónica Teoría de la Educación. \\
Educación y Cultura en la Sociedad de la Información.
\end{tabular}

datos”, etc. Se argumentará que sin la metodología comunicativa crítica difícilmente podríamos abordar con éxito el estudio y análisis de problemas educativos y sociales de máxima actualidad, con garantías de éxito. También se discutirá la idea de la neutralidad de la propia investigación y el objetivo de esta misma, desde el punto de vista de la transformación (o no) de la realidad de estudio.

\section{2.- LA METODOLOGÍA COMO HERRAMIENTA DE COMPRENSIÓN DE LOS FENÓMENOS SOCIALES Y EDUCATIVOS ACTUALES}

El International Congress of Qualitative Inquiry es uno de los espacios de discusión actuales más respetados por la comunidad científica internacional, donde se discuten y se presentan metodologías de investigación en ciencias sociales y educativas. Es un lugar donde pueden verse muy diversas tendencias metodológicas a nivel cualitativo que se están aplicando actualmente para realizar investigaciones en todo el mundo.

En los últimos años, cada vez están ganando más terreno aproximaciones tales como el enfoque de Social Justice, las autoetnografías y los estudios de representaciones (performance studies), la perspectiva crítica, las metodologías de carácter democratizador, la indagación narrativa, el enfoque de las Indigenous Pedagogies, entre otras muchas. Como se puede apreciar, las metodologías actuales por un lado cuestionan la noción clásica de investigación como un acto realizado de manera unívoca por el investigador (o el equipo de investigadores), que analizan la realidad (los datos) desde su particular visión de la realidad, usando para ello las debidas precauciones para evitar sesgos que condicionen (y comprometan) la veracidad y/o fiabilidad de las conclusiones a las que llegan. El binomio objetivismo/subjetivismo ya ha sido ampliamente superado, cuando ha quedado patente que una metodología basada en pretensiones de poder en las que se legitima el investigador para justificar la permanencia de su punto de vista por encima de interpretaciones de personas ajenas al ámbito académico no contribuye al desarrollo del conocimiento, sino que lo dificulta (CREA, 2001-2004).

Las aproximaciones metodológicas actuales crean mecanismos para incluir (de manera controlada, en términos científicos) las voces de todas las personas involucradas en el acto de investigar. El "giro dialógico" del que hablábamos en el apartado anterior se materializa también en el ámbito de la investigación en esta tendencia hacia la inclusión de las voces y el aumento del diálogo generador de conocimiento.

Pero, por otro lado, también existe un debate ético sobre el propio alcance de la investigación, que es una discusión que viene de lejos, sobre todo de la crítica que desde la antropología se hizo especialmente durante la segunda mitad del siglo pasado a los estudios de marcado carácter imperialista (occidentalista). Estos estudios estaban orientados por la exclusión de la voz de las comunidades de estudio. El resultado fueron detallados estudios que no hacían sino describir las comunidades objeto de investigación. Y, además, eran descritas desde los parámetros occidentales. Los debates sobre la validez de tales investigaciones dieron lugar a la revisión de técnicas como la etnografía, desde un paradigma nuevo (en aquel momento), como era la aproximación socio crítica. Más

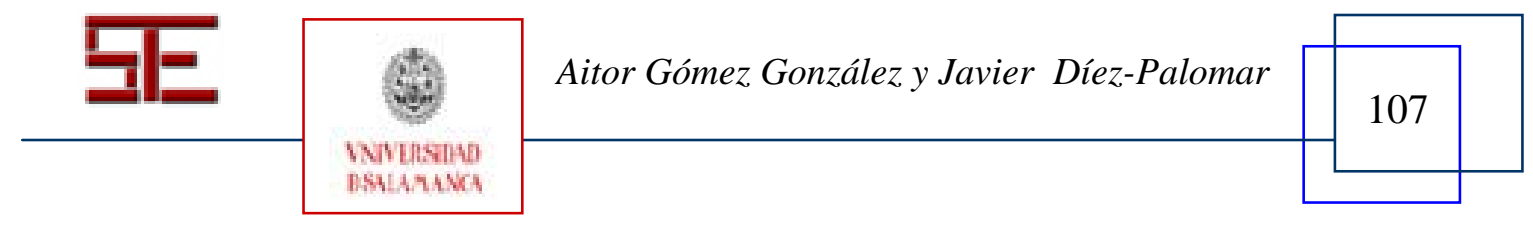


adelante, durante los años setenta, y sobre todo en la década de los ochenta, el constructivismo apareció junto a la perspectiva socio crítica, como una crítica relativista que matizaba el discurso clásico de investigación (y también la perspectiva socio crítica). Enfoques como la investigación-acción, la indagación, el interaccionismo simbólico, la etnometodología, pasan a ocupar las primeras páginas de la discusión metodológica sobre cómo hacer investigación y no caer en manipulaciones de la realidad. El "giro lingüístico” de las ciencias sociales, y un momento histórico quizás caracterizado por una gran incertidumbre y falta de referentes ante un cambio que no se sabía hacia dónde iba, crearon un marco de indeterminación que fue ocupado en buena parte por aproximaciones relativistas y teorías postmodernas muchas veces incluso abiertamente acientíficas que poco o nada tenían que aportar a la labor investigadora.

Actualmente el debate sobre la validez y la fiabilidad de las herramientas que utilizamos para hacer investigación continúa. El marco actual es un mundo preocupado por las desigualdades, por las formas de exclusión, por la globalización, la falta de representación de comunidades y colectivos "minoritarios" en los órganos de decisión de nuestras sociedades. Y además, como hemos visto, es un mundo donde el acceso a la información es cada vez más universal. En este marco, las metodologías no pueden quedarse al margen, si quieren contribuir con aportaciones que generen conocimiento que nos permita entender y comprender mejor la sociedad en la que vivimos. Por supuesto, el debate ético continúa: ¿debe la investigación involucrarse y transformar las situaciones de desigualdad? ¿Debe quedarse como un mero espectador de lo que está ocurriendo a nuestro alrededor?

La metodología comunicativa crítica es una aproximación que ofrece una posible respuesta a esta situación de cambio social. Incorpora las voces de todas las personas involucradas en la investigación desde el inicio hasta el final de la misma. Crea conocimiento científico a través de validar tanto el discurso de los expertos, como el discurso de las personas no expertas. La validación (y por tanto, la fuente de legitimación) del conocimiento son los argumentos contrastados en base a pretensiones de veracidad, no por la posición de poder de quien presenta tales argumentos. La interpretación sobre un fenómeno social o educativo de una persona ajena al mundo académico es tan válida como la explicación técnica de un profesor de universidad; si y sólo si ambas están regladas por pretensiones de veracidad. No tiene más razón quien mejor utiliza las estrategias y recovecos del discurso para crear un lenguaje tecnicista pero vacío de contenido y ajeno a la realidad que quiere interpretar. Desde la perspectiva de la metodología comunicativa crítica, el conocimiento es resultado de un diálogo que incorpora todos los saberes y puntos de vista de toda la comunidad. En un mundo global, abierto a la comunicación, donde existen tantos detalles, tantas complejidades, la única manera de acrecentar nuestro conocimiento es trabajar conjuntamente, compartiendo de manera solidaria nuestros saberes, nuestras formas de hacer, en base a una serie de criterios comunes, y con el objetivo de trabajar por el aumento del conocimiento, y no otras finalidades que nada tienen que ver con la ciencia.

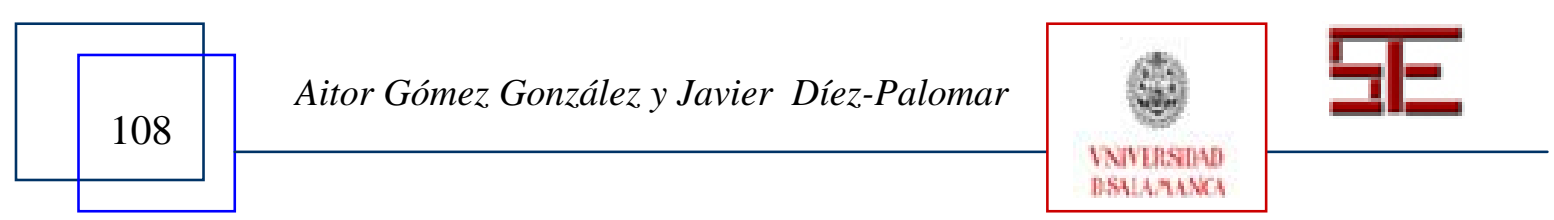




\begin{tabular}{c|c} 
Revista Electrónica Teoría de la Educación. \\
Educación y Cultura en la Sociedad de la Información.
\end{tabular}

\section{3.- FUNDAMENTOS DE LA METODOLOGÍA COMUNICATIVA CRÍTICA}

La metodología comunicativa crítica es una perspectiva metodológica que toma como uno de sus pilares más importantes el hecho de que el conocimiento no es en ningún caso patrimonio de las personas "expertas". La veracidad y validez de los resultados obtenidos a través de la investigación en las ciencias sociales dependen, en buena medida, de la capacidad de la perspectiva metodológica utilizada para integrar la intersubjetividad (Gómez, Latorre, Sánchez y Flecha, 2006). El concepto que subyace a esta afirmación es la idea de racionalidad comunicativa. Siguiendo las aportaciones que ha realizado Habermas (2001) al desarrollo de las ciencias sociales y educativas, la racionalidad de las acciones y de los procesos sociales no se mide únicamente bajo criterios instrumentales, tal y como habían destacado autores como Weber ${ }^{2}$. También existen otros elementos, como es el caso de la racionalidad comunicativa, basada en los acuerdos a los que se llega mediante el diálogo que se establece entre dos o más personas, con la idea de alcanzar un consenso.

Desde el punto de vista epistemológico, esta perspectiva implica asumir que los enunciados científicos son resultado del diálogo. El conocimiento, como tal, no es ajeno a las personas que lo hemos creado. Pero más allá de la visión constructivista de creación de saber, la metodología comunicativa crítica se centra en el aspecto intersubjetivo de la creación de conocimiento: todo lo que forma parte de nuestro saber es producto de la labor y el trabajo de generaciones y generaciones de personas que en el pasado hicieron aportaciones que han acabado formando parte del conocimiento que hoy día tenemos del mundo que nos rodea. Estas aportaciones nunca, en ningún caso, son producto de una sola persona. Al contrario: son resultado de muchas personas que mediante el diálogo, la discusión, la reflexión, el intercambio de ideas, en base a argumentos y evidencias han justificado y demostrado sus afirmaciones y han pasado a formar parte de nuestra visión del mundo. Tal y como dijo Kuhn en La estructura de las revoluciones científicas, los paradigmas son producto de acuerdos históricos, que van cambiando a lo largo del tiempo, cuando aparecen suficientes evidencias que justifican el cambio de paradigma (Kuhn, 1971). La idea de "diálogo en base a argumentos que se rigen por pretensiones de validez y no pretensiones de poder" es un aspecto clave que se destaca desde la metodología comunicativa crítica, porque los enunciados no se asumen por la posición de poder de quien los emite, sino por la corrección (y ajuste a la realidad) de los argumentos que se presentan para justificarlos. Partiendo de esta idea, esta aproximación metodológica es profundamente crítica con perspectivas basadas en el escepticismo, el relativismo epistemológico y moral o el nihilismo intelectual. Lejos de afirmar que la ciencia no es posible, y que todo consiste en la capacidad que tenga el investigador para imponer su punto de vista en base a la posición de poder que ocupa, desde la perspectiva comunicativa crítica se afirma que sí es posible hacer ciencia, construir enunciados que son verdaderos o falsos (que, por tanto, están sujetos a la posibilidad de falsación), y que lo que importa son las evidencias que se aportan para justificarlos (o rechazarlos).

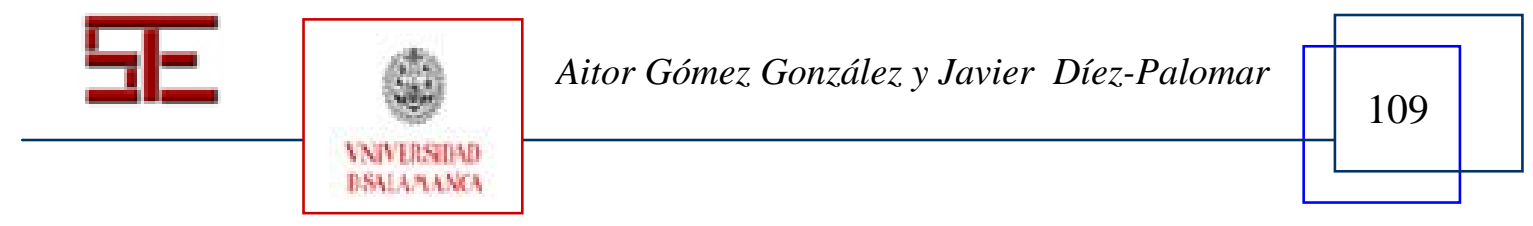


La metodología comunicativa crítica se asienta sobre una serie de postulados presentados ampliamente en Gómez, Latorre, Sánchez y Flecha (2006). Las investigaciones que se han hecho en el pasado sobre diversos ámbitos de la realidad social y educativa por lo general o bien se centraban en los individuos (sujetos) y en su capacidad de agencia para actuar (o no); o bien ponían el énfasis en el análisis de las estructuras, sus elementos deterministas, y el efecto que eso tiene (o no) sobre las acciones resultantes. Así, en la primera línea han destacado teorías como el interaccionismo simbólico, la fenomenología, la dramaturgia, etc. Cada cual con sus respectivas aproximaciones metodológicas (narrativa, [auto] biografía, etnografía, historia de vida, etc.). En cambio, en la segunda línea destacan el estructuralismo, el funcionalismo sistémico, etc. De la misma manera, también utilizan técnicas metodológicas propias (análisis del discurso, análisis semántico, semiótica, redes sistémicas, mapas conceptuales, etc.). La perspectiva comunicativa crítica se centra en un enfoque dual, que reconoce la capacidad de transformación de las personas sobre los acontecimientos de su entorno, pero por otro lado también tiene presentes las constricciones de las estructuras que condicionan el margen de actuación de las personas (tal y como han analizado autores como Chomsky, 1988; Searle, 2001; Habermas; 2001, y Beck, 1998). Los postulados sobre los que se asienta esta perspectiva son los siguientes:

- Universalidad del lenguaje y de la acción. La metodología comunicativa crítica se asienta en la idea de que todas las personas tenemos competencias lingüísticas comunicativas. Tal y como decía Habermas (2001) todas las personas somos sujetos capaces de lenguaje y acción. Por el hecho de ser "seres humanos", las personas tenemos dicha capacidad inherente a nuestra propia naturaleza. Searle (1997) destaca que debemos usar tipos de lenguaje que nos permitan alcanzar procesos de entendimiento que eliminen las distorsiones de la comunicación. Austin (1971), por ejemplo, propone los conceptos de "actos de lenguaje perlocucionarios" y "actos [de lenguaje] ilocucionarios" para referirse a cómo usamos el lenguaje para conseguir imponer nuestra opinión, o transmitir una idea o conocimiento. Por otro lado, Vygostky (1996) destaca por un lado la capacidad del lenguaje como catalizador de nuestro conocimiento transmitido de generación en generación, y por otro la característica del lenguaje como herramienta para resolver problemas a nivel cognitivo. También reflexiona sobre la importancia de la cultura y demuestra que no hay destrezas superiores o inferiores según el grupo cultural; hay destrezas diferentes. Investigaciones de Luria (1987), Cole y Scribner (1977), Lave y Wenger (1991), entre muchos otros, demuestran que las capacidades cognitivas y las habilidades comunicativas dependen del contexto, no de la disposición cognitiva innata de la persona. Incluso las personas más desfavorecidas, con menos oportunidades de acceder a espacios de conocimiento (como pueda ser la escuela), desarrollan estrategias en dichos contextos. Por tanto, el diálogo en términos de igualdad es necesario para aprehender toda esa diversidad y todo ese conocimiento. Por ejemplo, el saber sobre las principales reglas de la aritmética que tienen los niños que venden por las calles de Brasil es de un nivel increíblemente alto; pero ese código no está reconocido (ni legitimado) en la escuela, de manera que esos mismos niños que saben encontrar todas las estrategias para obtener beneficios en sus negocios no logran obtener buenas calificaciones en

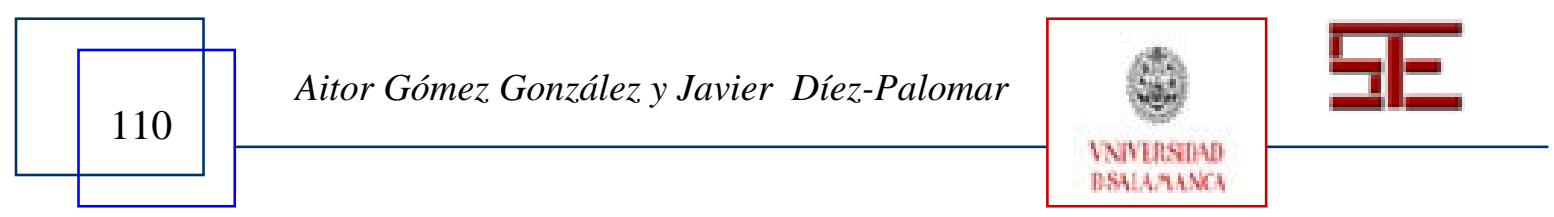




\begin{tabular}{c|c} 
Revista Electrónica Teoría de la Educación. \\
Educación y Cultura en la Sociedad de la Información.
\end{tabular}

la escuela (Carraher, Carraher y Schliemann, 1982). Desde la perspectiva comunicativa crítica todas estas capacidades y habilidades también forman parte de la aritmética. El diálogo igualitario sirve para incluir sus voces en la creación de dicho conocimiento.

- Las personas como agentes sociales transformadores. Las teorías sistémicas sostienen que las estructuras generan una serie de procesos que tienden (de manera funcional) a mantener la continuidad de dicha estructura y hacerla perdurar en el tiempo. Utilizando la analogía del "ser vivo", las estructuras generan una serie de comportamientos que tienden a reproducirlas a lo largo del tiempo. Sin embargo, el cambio social existe, ha habido sistemas que han aparecido y desaparecido a lo largo de la historia. Esto es debido a la capacidad de transformación de las personas. Tal y como argumenta Garfinkel (1967), las personas no somos idiotas culturales. Interpretamos la realidad social, creamos conocimiento nuevo y transformamos el existente. Desde el punto de vista de la filosofía de la ciencia (en términos epistemológicos) esto quiere decir que los enunciados científicos y las teorías no están dados de una vez para siempre, sino que van cambiando a medida que aumentamos el conocimiento de nuestro entorno (ya sea por avances técnicos que nos facilitan instrumentos que nos dejan profundizar en la visión que tenemos de las cosas, y por tanto en nuestra experiencia empírica; o ya sea porque nuevos constructos lógico-deductivos vienen a refutar afirmaciones anteriores). Somos las personas quienes transformamos esos enunciados científicos. Desde la perspectiva comunicativa crítica, además, se sostiene que la incorporación de más voces al debate científico es una fuente de incremento de la experiencia empírica, y, por tanto, de avance de la ciencia. Ejemplo de ello es el caso de la dimensión de "grupo étnico o cultural”, en antropología. Durante el siglo XIX y hasta mediados del siglo XX se trató a las comunidades indígenas allende de los mares como ejemplos de "comunidades humanas en niveles prehistóricos de desarrollo", que por tanto eran muestra de "cómo debió de ser la humanidad en sus primeros tiempos”. Análisis que han incluido las voces de las comunidades indígenas han dejado muy claro que no se trata de comunidades menos avanzadas, sino que tienen otros patrones culturales y se han desarrollado en otras direcciones, igualmente complejas desde el punto de vista cultural y tecnológico.

- La racionalidad comunicativa. Los desarrollos metodológicos de las ciencias sociales, hasta finales del siglo XX, habían pivotado sobre el concepto de racionalidad instrumental que acuñó Weber y que ha sido el referente durante más de un siglo. Habermas a finales de los años ochenta publicó La teoría de la acción comunicativa, donde no sólo analiza y reflexiona sobre el alcance de las categorías weberianas de acción, a la luz de la teoría de los clásicos de las ciencias sociales. También se da cuenta de que Weber no tuvo en cuenta otra forma de racionalidad, la comunicativa, que también da lugar a acciones sociales. Por tanto, la racionalidad comunicativa se convierte en otra de las grandes dimensiones de análisis, y las metodologías se ven en la necesidad de incluir dicha dimensión entre sus fundamentos epistemológicos. La metodología comunicativa crítica incluye dicha dimensión, a través de técnicas que sirven para analizar el diálogo intersubjetivo.

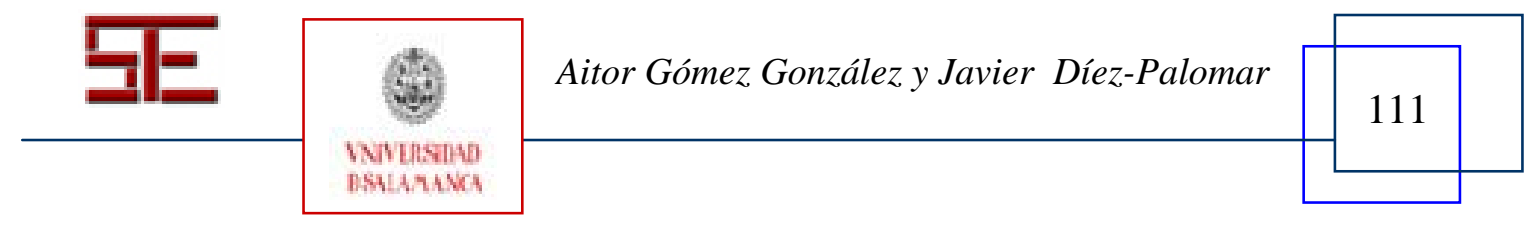


- El sentido común. Gramsci ya apuntaba en sus obras la necesidad de revisar el concepto de "saber científico" incluyendo los conocimientos que la gente había adquirido por métodos que se calificaban como no estrictamente académicos. En su obra legitimó como perfectamente válido todo conocimiento, viniera de la academia o de otra procedencia, pero siempre ajustado a unos criterios de verificación y de validez. Schütz (1993), por su lado, también realizó aportaciones a la misma idea, pero desde la línea de la fenomenología. Su teorización del sentido común en base a la experiencia previa de la vida y la conciencia de las personas constituyen un fundamento epistemológico que explica y justifica la necesidad de incluir todas las voces en el propio hecho de generación de conocimiento. La metodología comunicativa crítica, igual que otras líneas metodológicas actuales, incluye dicho elemento en sus bases de conocimiento.

- Sin jerarquía interpretativa. Dicho todo lo que venimos exponiendo en este artículo, queda claro otro de los supuestos interpretativos que subyacen a la metodología comunicativa crítica: la desaparición de la jerarquía interpretativa. Tal y como afirman Gómez, Latorre, Sánchez y Flecha (2006),

"los presupuestos interpretativos de las personas investigadas pueden tener tanta solidez como los del equipo investigador, de forma que éste no tiene por qué otorgarse el papel de intérprete científico: los individuos y las sociedades tienen capacidad para interpretar y comprender el mundo social" (p. 43).

Eso quiere decir que la validez de las interpretaciones depende no de la posición de poder que ocupe quien las realiza, dentro de la jerarquía interpretativa, sino de la validez de los argumentos que pone sobre la mesa.

- Igual nivel epistemológico. Otro elemento clave es que todas las personas tienen que tener la misma oportunidad de interpretar la realidad social. Si la jerarquía interpretativa desaparece en pro de argumentos basados en criterios de validez y veracidad, eso quiere decir que se necesita una metodología que sea capaz de crear espacios reales para que dicha posibilidad de interpretación sea real. Aunque realmente es difícil de alcanzar esta igualdad, cuando se consigue, las interpretaciones se enriquecen de las aportaciones tanto de la experiencia (expertice) de los científicos, como de la experiencia vivida de las personas que forman parte de la realidad social que estamos analizando.

- Conocimiento dialógico. Finalmente, todos estos supuestos giran en torno a la idea de que existe la posibilidad de conocimiento dialógico del mundo social. Habermas (2001) ya puso las bases teóricas de esta concepción epistemológica. Vygostky (1995) también estudió y mostró cómo el conocimiento es resultado de la interacción con el entorno. Una interacción que no es neutral (Freire, 1970), sino resultado de un diálogo entre ciencia y sociedad, como afirman Gómez, Latorre, Sánchez y Flecha (2006), en base al trabajo realizado por CREA durante más de dos décadas de investigación social y educativa de alto nivel. La metodología comunicativa crítica integra en su base epistemológica y de métodos esta dimensión del conocimiento, como un producto social, que inte-

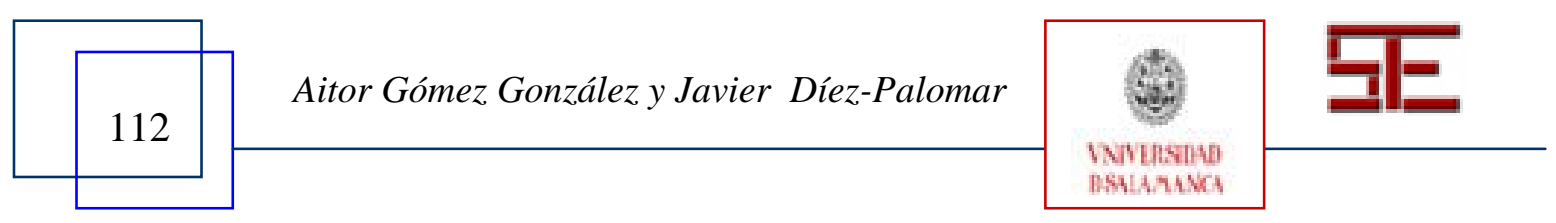




\begin{tabular}{c|c} 
Revista Electrónica Teoría de la Educación. \\
Educación y Cultura en la Sociedad de la Información.
\end{tabular}

gra postulados científicos, pero también experiencia de la vida. Es el resultado de procesos tanto deductivos, como inductivos, tanto empiristas, como lógico deductivos. Por ese motivo, supone una contribución hacia el conocimiento en las modernas sociedades del siglo XXI.

\section{4.- METODOLOGÍA COMUNICATIVA CRÍTICA EN PROYECTOS DE IN- NOVACIÓN Y DESARROLLO INTERNACIONALES}

Como ya hemos plasmado, la metodología comunicativa crítica, basada en el giro dialógico que se está produciendo en nuestras sociedades (Gómez et al., 2006), posee una amplia y diversa base teórica que analiza cómo la sociedad se torna cada vez más dialógica. Una de las principales características de la metodología comunicativa crítica es su compromiso con la superación de las desigualdades sociales, que se consigue relacionando las principales teorías en ciencias sociales y las propuestas transformadoras que surgen de los agentes sociales. Es decir, aunando sistema y mundo de la vida y desmarcándose de otras concepciones que sólo tienen en cuenta a los sujetos (etnometodología, interaccionismo) y aquellas que sólo tienen presente a las estructuras (funcionalismo, estructuralismo, postestructuralimo).

De esta forma, la metodología comunicativa crítica rompe con el desnivel cualitativamente relevante (Habermas, 2001) existente entre sujetos y sistema, potenciando que tanto actores sociales como investigadores e investigadoras participen, a través de un diálogo igualitario, desde el inicio hasta el final de la investigación. La pluralidad de voces, atendiendo a los mejores argumentos aportados por cada persona participante, facilita no caer en visiones sesgadas de la realidad en la que en ocasiones caemos las y los investigadores al no relacionar lo que nos aporta el conocimiento científico con las argumentaciones de los actores sociales.

A nivel educativo, por ejemplo, no es sencillo encontrar centros donde los y las profesionales potencien el éxito escolar para todo el alumnado, llegándose a relativizar la importancia que tiene orientar a las y los niños hacia el bachillerato y universidad. Al no haber diálogo entre todos los sujetos que intervienen en el proceso educativo, partiendo de las principales contribuciones de la comunidad científica internacional, se llegan a potenciar itinerarios educativos diferenciados que llevan al alumnado más necesitado fuera del aula ordinaria y después fuera del centro.

El desconocimiento de lo que la comunidad científica internacional lleva ya más de dos décadas demostrando, que los grupos homogéneos llevan al fracaso escolar, junto con la falta de diálogo con los actores sociales lleva a profesionales de la educación a potenciar este tipo de prácticas segregadoras. El diálogo plural potenciado por la metodología comunicativa crítica (Flecha y Gómez, 2004) proporciona argumentos que cuestionan esas prácticas y abren vías de superación de las desigualdades educativas y sociales.

A través del proyecto integrado INCLUD-ED (2006-2011) ${ }^{3}$ se ha constatado a nivel teórico y práctico como la implicación de las familias y la comunidad en los procesos

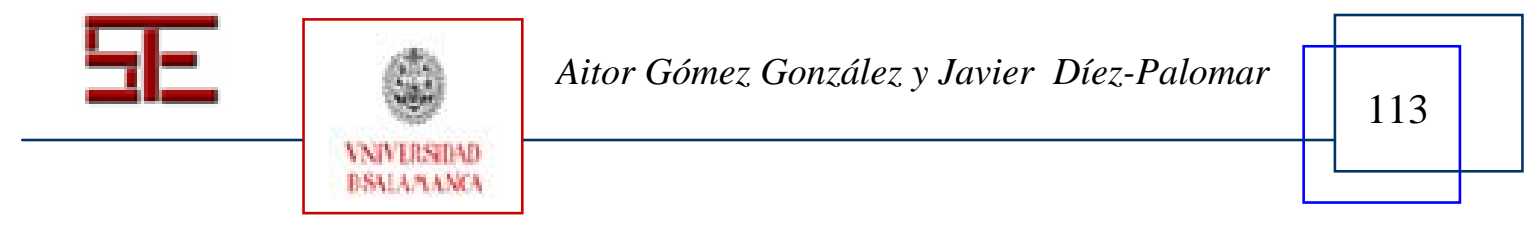


educativos del alumnado mejora su rendimiento académico. En el proyecto 6 se han llevado acabo estudios de caso utilizando metodología comunicativa crítica. Se han analizado cuatro formas diferentes de participación de familiares y la comunidad ${ }^{4}$ dentro de los centros escolares, teniendo impacto positivo todas ellas en los resultados académicos del alumnado, llevándose a cabo en contextos de bajo nivel socioecómico.

Al hacer partícipes de los procesos de toma de decisión, de participación en las aulas y otros espacios de aprendizaje y llevar a cabo una formación dirigida a familiares que parte de sus necesidades y también competencias, se logran mejores resultados académicos de todo el alumnado. La metodología comunicativa crítica utilizada para llevar a cabo los estudios de caso ha permitido obtener unos resultados que de otra manera no hubieran sido posibles. Se han identificado las principales barreras que dificultan la participación de familiares en los procesos educativos de sus hijos y de manera paralela se han apuntado los elementos que propician éxito educativo para todo el alumnado.

$\mathrm{Al}$ revisarse las principales teorías al respecto y relacionarlas con las informaciones obtenidas fruto de la utilización de técnicas comunicativas de recogida de datos y disponer de datos cuantitativos sobre resultados académicos del alumnado en los últimos cinco años, se ha podido constatar que los centros estudiados que han llevado a cabo grupos interactivos dentro de las aulas y han potenciado la participación de familiares y comunidad en todas las dinámicas del centro han obtenido una mejora muy sustancial en los resultados académicos de su alumnado.

Los centros donde se han llevado a cabo los estudios de caso son comunidades de aprendizaje $^{5}$. En la fase de transformación de cualquier centro educativo en una comunidad de aprendizaje se cuenta con la participación de familiares y de toda la comunidad y en las diferentes comisiones de trabajo que se establecen también se cuenta con todos ellos y ellas. Es decir, participan en todas las esferas y desde el inicio del proceso. Esta forma de funcionamiento y de organización coincide plenamente con los principios y organización de una investigación que se lleve a cabo utilizando metodología comunicativa crítica. Se rompe con el desnivel cualitativamente relevante, desaparece la figura del experto, se potencian espacios de diálogo igualitarios y se toman decisiones de forma consensuada en base a los mejores argumentos aportados por familiares, voluntariado, alumnado o profesorado.

Los estudios de caso muestran además que la mejora de los resultados académicos se produce en unos centros educativos que están recibiendo un elevado número de alumnado inmigrante. Se incrementa año tras año el número de alumnado inmigrante y año tras año se van mejorando los resultados académicos, con lo cual, la crítica fácil que escuchamos a menudo relativa a que a mayor porentaje de alumnado extranjero en las aulas peores resultados académicos se obtienen no es cierta.

En el proyecto RTD Workaló (2001-2004) ${ }^{6}$ se llevaron a cabo unas jornadas finales donde se expusieron los principales resultados alcanzados durante los tres años de trabajo de investigación. En dichas jornadas intervinieron académicos de reconocido presti-

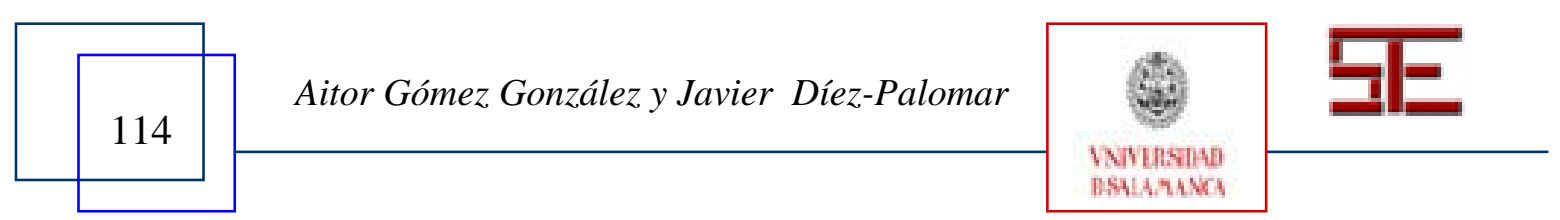




\begin{tabular}{c|c}
\hline 1 & $\begin{array}{c}\text { Revista Electrónica Teoría de la Educación. } \\
\text { Educación y Cultura en la Sociedad de la Información. }\end{array}$ \\
\hline & TESI, 10(3), 2009, 103-118
\end{tabular}

gio a nivel internacional que expusieron sus teorías. Uno de los expertos llevó a cabo su intervención sobre cómo estaba siendo conceptualizado el término mestizaje por parte de la comunidad científica internacional. Su argumentación giraba en torno a la idea de que las personas pertenecientes a grupos culturales no hegemónicos que viven en Europa son, por ejemplo, 50\% francesas y 50\% árabes. Una mujer perteneciente a una minoría étnica que se encontraba en la sala puntualizó la afirmación del experto diciendo que "no soy $50 \%$ francesa y $50 \%$ gitana, sino $100 \%$ francesa y $100 \%$ gitana". La mujer prosiguió con su argumentación afirmando que "cuando tú votas en las elecciones francesas tu voto vale 1 , cuando voto yo ¿qué vale?, ¿sólo el $50 \%$ de 1 , sólo medio voto? El mío vale 1, igual que el tuyo porque soy igual de francesa que tú, 100\% francesa y también soy $100 \%$ gitana, $100 \%$ mujer y 100\% también de otras identidades”. El experto afirmó después de esta intervención que "tendremos que replantearnos el concepto científico de mestizaje”. Intervenciones como las llevadas a cabo por esta mujer, en espacios ideales de habla, generan que nos replanteemos nuestros conceptos y podamos mejorarlos.

La metodología comunicativa crítica utilizada en el proyecto Workaló fue, lógicamente, también utilizada a la hora de concretar las jornadas finales. De esta forma, se creó un espacio donde se rompía con la diferenciación entre "sujeto investigador" y "objeto investigado" y las personas expertas no ejercían ese rol, sino que aportaban su conocimiento y entraban a dialogar en un plano de igualdad con todas las personas asistentes. Fruto de ese planteamiento es posible que consigan elaborar teorías de elevada calidad científica, ya que se conjuga el saber académico con las aportaciones de las personas participantes.

\section{5.- A MODO DE CONLUSIÓN}

La metodología comunicativa crítica tiene como uno de sus pilares básicos el partir de los conocimientos científicos previos para que las investigaciones llevadas a cabo sean de calidad científica y a la vez útiles socialmente. Como investigadores e investigadoras tenemos la responsabilidad de aportar ese conocimiento científico acumulado durante el desarrollo de nuestras investigaciones. Las personas investigadas aportan sus argumentos y se incorporan a ese bagaje científico ya existente. Las resistencias ejercidas por los representantes del sistema científico argumentando que el sentido común aportado por los sujetos sociales no puede ser considerado como científico han repercutido de manera muy negativa en el desarrollo de la ciencia. Estos científicos, al estar mediatizados por relaciones jerárquicas, priorizan el entendimiento con personas con niveles de estudios elevados a hacerlo con personas con escasos estudios.

La metodología comunicativa crítica, al partir del conocimiento científico ya existente, recoge aportaciones de los paradigmas objetivista, subjetivista y sociocrítico, sin incurrir en sus errores. Se priorizan los conocimientos científicos acumulados coincidiendo en este sentido con las metodologías objetivistas de investigación y alejándose de las subjetivistas. Pero a la vez de una importancia vital a las voces de los sujetos sociales, punto en el cual coincide plenamente con metodologías como la investigación-acción y

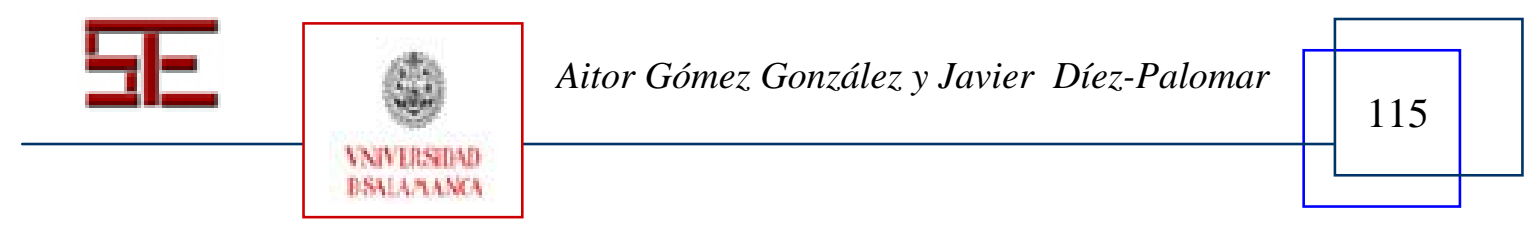


la investigación participativa del planteamiento subjetivista y difiriendo en este caso del objetivista. Desde el planteamiento comunicativo crítico ese conocimiento acumulado no es propiedad de la comunidad científica, sino de todas las personas.

La base para conseguir un incremento de la utilidad social de los resultados de la investigación y una mayor calidad científica es el diálogo igualitario entre las personas investigadas e investigadoras. Este diálogo es igualitario porque se priorizan los argumentos de las personas que intervienen y no la posición académica o social que ocupen. La construcción del conocimiento científico se lleva a cabo a través de la aportación de una gran diversidad de argumentaciones que no atienden a jerarquías ni imposiciones de las opiniones de personas “expertas”.

La metodología comunicativa es una seria alternativa a las investigaciones tradicionales que no pueden proporcionar los conocimientos necesarios para analizar y superar problemas como por ejemplo la convivencia entre adolescentes. A través de esta metodología obtenemos una base sólida de conocimientos para poder plantear con éxito medidas que ayuden en la mejora de la convivencia y de esta forma se puedan transformar situaciones de desigualdad y discriminación por cuestiones de género o etnia.

\section{6.- BIBLIOGRAFÍA}

AUBERT, A.; FLECHA, A.; GARCÍA, C.; FLECHA, R. y RACIONERO, S. (2008): Aprendizaje dialógico en la Sociedad de la Información. Barcelona, Hipatia Editorial S.A.

AUSTIN, J. L. (1971): Cómo hacer cosas con palabras. Palabras y Acciones. Buenos Aires, Paidós.

BECK, U. (1998): La sociedad del riesgo. Barcelona, Paidós.

BECK, U.; GIDDENS, A. y LASH, S. (1997): Modernización Reflexiva. Política, transición y estética en el orden social moderno. Barcelona, Península.

CARRAHER, T. N.; CARRAHER, D. W. Y SCHLIEMANN, A. D. (1982): Na vida, dez, na escola, zero: os contextos culturais da aprendizagem da matematica, $\mathrm{Ca}$ dernos de Pesquisa, 42. 79-86.

CHOMSKY, N. (1988): Language, mind and politics. New York: Black Rose Books.

COLE, M. Y SCRIBNER, S. (1977): Cultura y pensamiento. Relación de los procesos cognoscitivos con la cultura. México, Limusa.

CREA (2001-2004): Workaló. The creation of new occupational patterns for cultural minorities: The Gypsy Case. Proyecto para el V programa marco de la Unión Europea.

FLECHA, R. y GÓMEZ, J. (2004): Participatory Paradigms: Researching 'with' rather than 'on', en CROSSAN, B.; GALLACHER, J. y OSBORNE, M. (eds.) Researching Widening Access: Issues and approaches in an international context. London, Routledge, 129-140.

FLECHA, R.; GÓMEZ, J. Y PUIGVERT, L. (2001): Teoría sociológica contemporánea. Barcelona, Paidós.

FREIRE, P. (1970). La pedagogía del oprimido. Madrid, Siglo XXI.

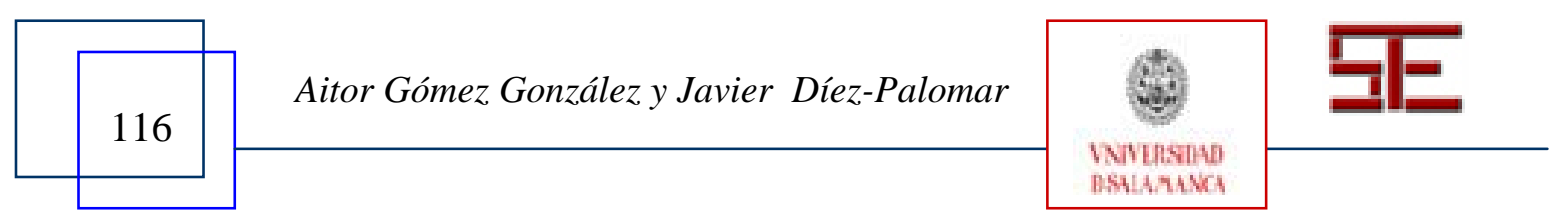




\begin{tabular}{c|c}
\hline 1 & $\begin{array}{c}\text { Revista Electrónica Teoría de la Educación. } \\
\text { Educación y Cultura en la Sociedad de la Información. }\end{array}$ \\
\hline & TESI, 10(3), 2009, 103-118
\end{tabular}

GARFINKEL, H. (1967). Studies in Ethnomethodology. Englewwod-Cliffs (N. J.), Prentice Hall.

GIDDENS, A. (1995): Modernidad e identidad del yo. Barcelona, Península.

GÓMEZ, J.; LATORRE A.; SÁNCHEZ, M. y FLECHA, R. (2006): Metodología Comunicativa Crítica. Barcelona, El Roure editorial.

HABERMAS, J. (2001): Teoría de la acción comunicativa. Volumen I: Racionalidad de la acción y racionalización social y Volumen II: Crítica de la razón funcionalista. Madrid, Taurus.

KUHN, T. (1971). La estructura de las revoluciones científicas. México, Fondo de Cultura Económica.

LAVE, J. Y WENGER, E. (1991): Situated Learning. Legitimate peripheral participation. Cambridge, MA, Cambridge University Press.

LURIA, A, R. (1987): Desarrollo histórico de los procesos cognitivos. Madrid, Akal.

SCHÜTZ, A. (1993): La construcción significativa del mundo social. Barcelona, Paidós.

SEARLE, J. (1997): La construcción de la realidad social. Barcelona, Paidós.

--(2001): Mente, lenguaje y sociedad. La filosofía en el mundo real. Madrid, Alianza.

VYGOTSKY, L. S. (1995): Pensamiento y lenguaje. Barcelona, Paidós.

-- (1996): El desarrollo de los procesos psicológicos superiores. Barcelona, Crítica.

\section{Notas}

${ }^{1}$ Para una reflexión sobre las consecuencias de este cambio social, ver Giddens Modernidad e identidad del yo. En este libro Giddens describe los cambios sociales que se están produciendo en lo que él llama "segunda modernidad”, y el impacto que tienen sobre la propia definición que cada persona hace de sí misma. La existencia de un gran abanico de posibilidades de elección provoca que tomemos "riesgos" (por eso otros autores hablan de sociedad del riesgo) al tomar una de ellas, y desestimar el resto. Estos riesgos vienen a veces acompañados también de una situación de angustia existencial, que nos acompaña y forma parte de nuestra identidad.

${ }^{2}$ Cuando Weber reflexiona sobre el concepto de "racionalidad" distingue entre las acciones teleológicas (instrumentales) con arreglo a fines o a valores. También habla de las acciones sociales tradicionales y afectivas, que separa de las racionales. Esta discusión ha marcado la sociología y el desarrollo de la investigación social durante más de un siglo, hasta que Habermas ha logrado hacer una revisión de las categorías weberianas para proponer un nuevo esquema que va más allá del análisis de la racionalidad como un elemento únicamente instrumental, añadiendo el punto de vista comunicativo, basado en la acción sujeta al consenso de los actores. Para ampliar este punto de vista, ver Habermas (2001).

${ }^{3}$ INCLUD-ED. Strategies for inclusion and social cohesion from education in Europe. INTEGRATED PROJECT Priority 7 of Sixth Framework Programme (2006- 2011) (para más información visitar el siguiente sitio web: http://www.ub.es/includ-ed/).

${ }^{4}$ Las cuatro formas de participación analizadas han sido: formación de familiares, participación en los procesos de toma de decisión, participación en las aulas y otros espacios de aprendizaje y participación en el desarrollo del currículum y en la evaluación.

${ }^{5}$ El proyecto de Comunidades de Aprendizaje es un proyecto de cambio en la práctica educativa para responder de forma igualitaria a los retos y necesidades que se plantean en la sociedad actual. Extraído el 2 de mayo de 2009 de: http://www.utopiadream.info/red/tikidownload_wiki_attachment.php?attId=Presentaci\%C3\%B3n.

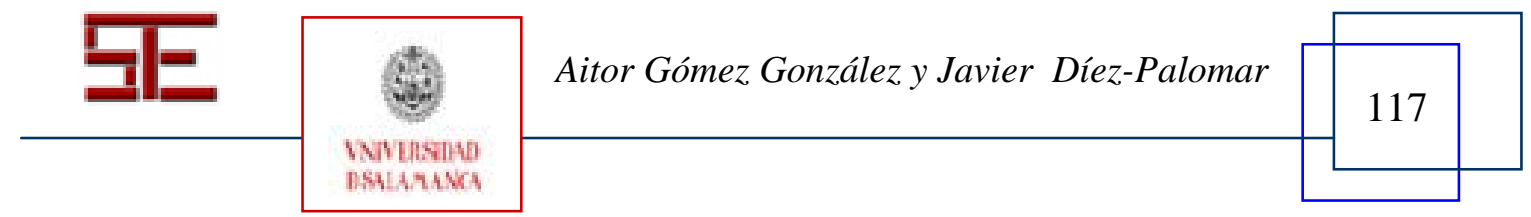


Revista Electrónica Teoría de la Educación.

Educación y Cultura en la Sociedad de la Información.

TESI, 10(3), 2009, 103-118

${ }^{6}$ WORKALÓ. The creation of New occupational patterns for cultural minorities. The Gypsy case. RTD. FP5. DG XII. Improving the Socio-economic Knowledge Base (2001-2004) (para más información consultar el siguiente sitio web: http://www.neskes.net/workalo/).

Para citar el presente artículo puede utilizar la siguiente referencia:

GÓMEZ GONZÁLEZ, A. y DÍEZ-PALOMAR, J.: (2009): Metodología comunicativa crítica: transformaciones y cambios en el S. XXI, en FLECHA GARCÍA, R. y STEINBERG, S. (Coords.) Pedagogía Crítica del S. XXI [monográfico en línea]. Revista Electrónica Teoría de la Educación: Educación y Cultura en la Sociedad de la Información. Vol. 10, $\mathrm{n}^{0}$ 3. Universidad de Salamanca, pp. 103-118 [Fecha de consulta: $\mathrm{dd} / \mathrm{mm} /$ aaaa $]$.

http://campus.usal.es/ revistas_trabajo/index.php/revistatesi/article/view/3964/3986

ISSN: 1138-9737

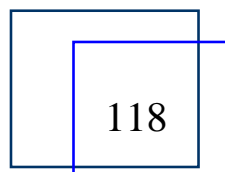

Aitor Gómez González y Javier Díez-Palomar

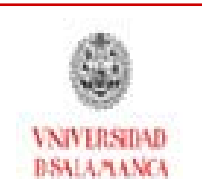

